\title{
Laser Radar based Vehicle Localization in GPS Signal Blocked Areas
}

\author{
Ming Yang \\ Department of Automation, Shanghai Jiao Tong University \\ Key Laboratory of System Control and Information Processing, Ministry of Education of China \\ Shanghai, 200240, China \\ Chunxiang Wang ${ }^{*}$, Hui Fang \\ Research Institute of Robotics, Shanghai Jiao Tong University \\ Shanghai, 200240, China \\ Bing Wang \\ Department of Automation, Shanghai Jiao Tong University \\ Key Laboratory of System Control and Information Processing, Ministry of Education of China \\ Shanghai, 200240, China \\ Received 5 July 2011; accepted 25 November 2011
}

\begin{abstract}
Reliable vehicle localization is a basic requirement in many applications in transportation field. GPS-based localization is quite popular nowadays. However, in urban environments applications, signal of GPS is often blocked by surrounding objects like high-rise buildings, tunnels, overhead roads, etc, making localization information unavailable. This paper proposed a laser radar based map matching approach to address this problem, especially when GPS signal blocked area is large. The proposed approach includes mapping and localization. In the mapping, after map initialization sensor data constraints are linearized to formulate an optimal linear estimation based map optimization framework, which can improve map accuracy effectively. In the localization, vehicle pose is estimated by matching the current laser scan with the best submap and by a UKF (Unscented Kalman Filter) based fusion strategy. Results from both synthetic and real experiments show good performance of the proposed approach.
\end{abstract}

Keywords: GPS Signal Blocked, Vehicle Localization, Mapping, Map Optimization

\section{Introduction}

Vehicle localization is a fundamental task in many applications $^{1}$. In-car navigation systems use its localization module to compute vehicle's current position and show it on a digital map to tell drivers how to get from one location to another efficiently. With a localization system, vehicle can communicate its position to a monitoring center, which will help operators to direct vehicle fleets as efficiently as possible since they know the traffic flow on different roads. Furthermore, vehicle localization is also quite important in intelligent transportation systems, such as
Advanced Driver Assistance Systems and driverless vehicles. They require reliable and accurate localization to make intelligent decisions or realize automatic navigation.

Global Positioning System (GPS) has been widely used in vehicle localization systems ${ }^{2,3}$. However, a big problem with GPS-based localization is that GPS signal is often blocked by surrounding objects, especially in urban environments ${ }^{4}$. For example, high-rise buildings, tunnels, overhead roads or even tall trees can block GPS signals seriously, making localization information unavailable. This is a quite critical problem, especially

\footnotetext{
*Corresponding Author: wangcx@sjtu.edu.cn

This work was financially supported by the National Natural Science Foundation of China (61174178) and the Shanghai Science and Technology Commission through Expo Science and Technology Program (10dz0581100)
} 
in applications requiring reliable and continuous vehicle localization.

Many efforts have been made to tackle this problem. Additional devices such as altimeters and precise clocks Ref.1 have been used to provide additional information. Ref.5 suggested a constrained method by modeling the traveling path as pieces of lines. In this method, the minimum number of satellites required is reduced to two. In Ref.6, authors used the constraints that the altitude of the vehicle and vehicle speed are approximately constant. Then they designed a localization algorithm requiring number of satellites fewer than 4. A more popular approach is the integration of GPS with Inertial Navigation Systems (INS) or dead reckoning sensors such as encoders to provide continuous localization information ${ }^{2}$. Although many approaches have been proposed, most of them assumed that the duration of the blockage of GPS signals is short (the GPS signal blocked area is small) or there is still several satellites can be viewed. How to localize a vehicle in a large blocked area without visible satellites? This is our motivation to carry out the work in this paper.

In large GPS signal blocked areas, map matching can be used to address the localization problem. It should be noted that the map matching here is quite different from the one often used in vehicle navigation systems, in which map matching is realized by matching the current vehicle location and history trajectory with the road network of a digital map ${ }^{7}$. In our case, map matching is carried out by matching sensor measurements with an environmental $\operatorname{map}^{8}$, which is often used in robotic field.

In map matching, one difficulty is how to create a precise map. Simultaneous Localization and Mapping $(\mathrm{SLAM})^{9}$ is an attractive technique for vehicle navigation in an unknown environment. However it may not be suitable for real-time accurate localization if it's applied in outdoor unstructured environments. Additionally, SLAM is not necessary for vehicle localization in urban environments (actually in GPS signal blocked areas) since the environments are known to us. Therefore, we can just first create a map and then use the map to localize a vehicle, which can ensure reliability and efficiency.

The proposed approach for vehicle localization in GPS signal blocked areas includes two steps: mapping and localization. Mapping is done offline and localization is real-time. In the mapping step, a vehicle is manually guided to explore environment (blocked area), recording sensor data from laser radar and odometry with time stamps. Additionally, GPS data in entrance and exit of blocked area is also recorded for mapping. Mapping algorithm utilizes these sensor data and an optimization strategy to create a precise map. Optimization is achieved based on linearizing constraints from sensor data. In the next step, the created map can be shared with other vehicles to localize themselves by map matching. Map matching applies a robust version of ICP (Iterative Closest Point) algorithm registering the current laser frame with the best submap. A UKF (Unscented Kalman Filter) based fusion strategy is also designed to improve reliability by fusing map matching result with dead reckoning.

The rest paper is organized as follows: section 2 firstly discusses the pairwise range scans registration problem, which is a fundamental technique in our mapping and localization approach; then section 3 describes how the recorded data is processed to create an accurate map. In section 4, a localization algorithm including map matching and fusion is presented. Next, section 5 shows experimental results with both synthetic and real data; finally, section 6 ends this paper with some conclusions.

\section{Pairwise Range Scans Registration}

Laser radar is used to collect environmental information in GPS signal blocked area. The laser radar was fixed on the top of the vehicle to reduce the effect of moving object. The output of laser radar is called range scan, which is a list of points corresponding to the intersection of a laser beam with objects in the environment. The laser beam rotates in a horizontal plane. Thus a range scan describes a $2 \mathrm{D}$ slice of the environment.

Both mapping and localization in the proposed approach are based on pairwise range scans registration. This technique is usually applied to compute translation and rotation between two frames to estimate ego motion of a vehicle. ICP is a useful algorithm for addressing the pairwise registration problem ${ }^{10}$. It selects the closest point as corresponding point and then estimates registration parameters by minimizing the value of a given error function. These two steps are iteratively implemented to obtain an accurate result. 
Establishing correct corresponding points is crucial in ICP. Here, we apply following three considerations for determining correspondences:

1) Corresponding points has shortest Euclidean distance in world reference frame. Furthermore this Euclidean distance has to be within the uncertainty of odometry data (odometry data is used to predict vehicle poses).

2) Corresponding points should have similar normal direction.

3) Applying M-estimator within iterative procedure.

Given $h$ pairs of corresponding points $\left\{\left(P_{k}, C P_{k}\right)\right\}$, $k=1,2 \ldots h$. Let be $(t x, t y, t r)$ the translation and rotation between two range scans. The objective of ICP is to estimate $(t x, t y, t r)$ accurately. The error function of ICP is defined in the form of weighted least-square:

$$
E_{\mathrm{ICP}}(t x, t y, t r)=\frac{1}{h} \sum_{k=1}^{h} \lambda_{k} \cdot\left(R_{t r} \cdot P_{k}+T-C P_{k}\right)^{2}
$$

where:

$$
\begin{aligned}
& P_{i}=\left[x_{k}, y_{k}\right]^{T} \quad C P_{k}=\left[c x_{k}, c y_{k}\right]^{T} T=[t x, t y]^{T} \\
& R_{t r}=\left[\begin{array}{cc}
\cos (t r) & -\sin (t r) \\
\sin (t r) & \cos (t r)
\end{array}\right]
\end{aligned}
$$

$\lambda_{k}$ is M-estimator coefficient, defined as:

$$
\lambda_{k}=\left\{\begin{array}{rr}
1, & \left|e_{k}\right| \leq \mu+\sigma \\
\sigma /\left|e_{k}\right|, & \mu+\sigma<\left|e_{k}\right| \leq \mu+3 \sigma \\
0, & \mu+3 \sigma<\left|e_{k}\right|
\end{array}\right.
$$

where $\left|e_{k}\right|$ is the absolute value of residual error, $\mu$ is the mean of $\left|e_{k}\right|, \sigma$ is the standard variance of $\left|e_{k}\right|$.

A closed-form solution can be derived as:

$$
\begin{aligned}
& t r=\arctan \frac{\sum_{k=1}^{h} \lambda_{k} \cdot\left[\left(x_{k}-\bar{x}\right)\left(c y_{k}-\overline{c y}\right)-\left(y_{k}-\bar{y}\right)\left(c x_{k}-\overline{c x}\right)\right]}{\sum_{k=1}^{h} \lambda_{k} \cdot\left[\left(x_{k}-\bar{x}\right)\left(c x_{k}-\overline{c x}\right)+\left(y_{k}-\bar{y}\right)\left(c y_{k}-\overline{c y}\right)\right]} \\
&\left\{\begin{array}{l}
t x=\overline{c x}-(\bar{x} \cdot \cos (t r)-\bar{y} \cdot \sin (t r)) \\
t y=\overline{c y}-(\bar{x} \cdot \sin (t r)+\bar{y} \cdot \cos (t r))
\end{array}\right.
\end{aligned}
$$

where:

$$
\begin{aligned}
& \bar{x}=\frac{\sum_{k=1}^{h} \lambda_{k} \cdot x_{k}}{\sum_{k=1}^{h} \lambda_{k}}, \bar{y}=\frac{\sum_{k=1}^{h} \lambda_{k} \cdot y_{k}}{\sum_{k=1}^{h} \lambda_{k}}, \\
& \overline{c x}=\frac{\sum_{k=1}^{h} \lambda_{k} \cdot c x_{k}}{\sum_{k=1}^{h} \lambda_{k}}, \overline{c y}=\frac{\sum_{k=1}^{h} \lambda_{k} \cdot c y_{k}}{\sum_{k=1}^{h} \lambda_{k}}
\end{aligned}
$$

The procedure of ICP algorithm is illustrated in Fig. 1. Odometry data is utilized to give initial values of ( $t x$, $t y, t r)$ to start ICP. Proper threshold need to be chosen to stop the iterative procedure according to the accuracy requirement. Pairwise registration technique will be applied both in mapping and localization. In the mapping, corresponding points determined in the final iterative step are used in map optimization. In the localization, it is used as basic map matching technique to estimate vehicle poses.

Pairwise registration may fail because of errors in sensor data acquisition and processing. If one of the following cases happens, the result of pairwise registration will be considered trustless:

1) The number of corresponding points established in final iterative step is smaller than a threshold (for example, 25).

2) The number of iterations exceeds the maximal one (for example, 30).

3) Vehicle displacement computed by pairwise registration is larger than a predicted uncertainty (by using odometry data).

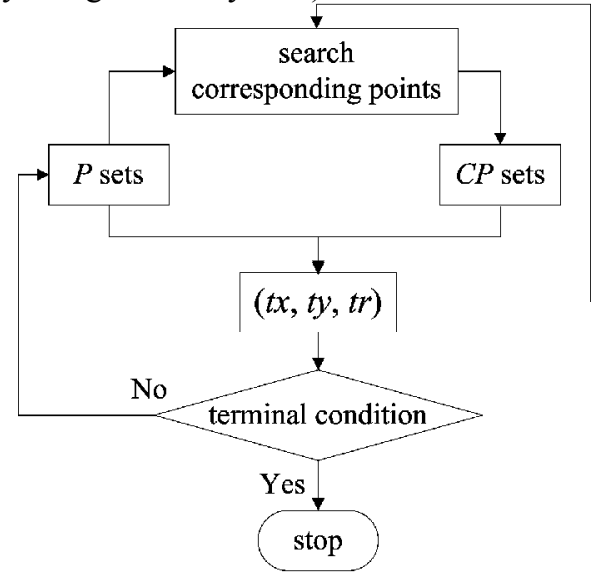

Fig. 1 Illustration of ICP algorithm

\section{Mapping in GPS Signal Blocked Area}

If the duration of GPS signals blockage is short, we can integrate dead-reckoning with GPS system to obtain a reliable localization. However, if the blockage duration is long, we have to apply other technique. Here, we apply map matching based localization. Thus, the first problem we need to solve is how to create a precise map.

\subsection{Coordinate Systems}

Fig. 2 shows the world reference frame and vehicle reference frame denoted by $\mathrm{X}_{\mathrm{W}} \mathrm{O}_{\mathrm{W}} \mathrm{Y}_{\mathrm{W}}$ and $\mathrm{X}_{\mathrm{V}} \mathrm{O}_{\mathrm{V}} \mathrm{Y}_{\mathrm{V}}$ respectively. The origin of vehicle reference frame is in the middle of the rear axle. Vehicle pose is denoted by $X$ 
$(x, y, r)$, representing position and orientation of a vehicle in the world reference frame.

Fig.3 illustrates the relationship between world coordinates $(x w, y w)$ and vehicle coordinates $(x v, y v)$ of a scan point $p$ (assume laser scan points have been transformed from laser coordinates to vehicle reference frame by calibration), which can be expressed as:

$$
\left[\begin{array}{l}
x w \\
y w
\end{array}\right]=\left[\begin{array}{cc}
\cos (r) & -\sin (r) \\
\sin (r) & \cos (r)
\end{array}\right] \cdot\left[\begin{array}{l}
x v \\
y v
\end{array}\right]+\left[\begin{array}{l}
x \\
y
\end{array}\right]
$$

Above equation can be rewritten as:

$[x w, y w]^{T}=f(x, y, r, x v, y v)=f(X, x v, y v)$

where $f$ is a non-linear coordinates transformation function.
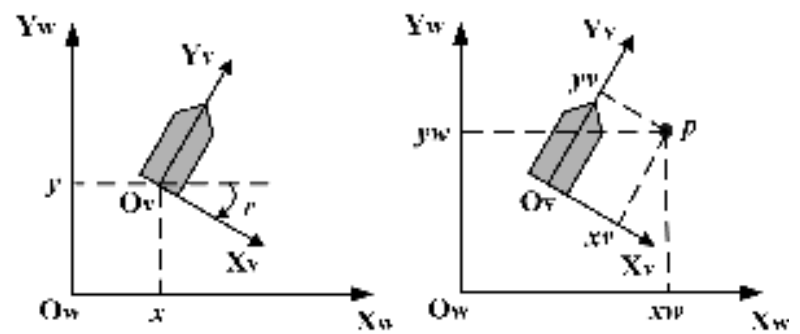

Fig. 2 Coordinates system Fig. 3 Coordinates relationship

\subsection{Scan Data Preprocessing}

Although laser radar has been fixed on the top of the vehicle aiming to reduce the effects of moving objects, we still need a special consideration on data preprocessing to extract stable environmental information to create a stable map. Some isolated points in the scan data have to been removed since they may be noises. And some unstable natural objects like tree leaves, which may reduce reliability in range scans registration, also need to be removed.

The first step of data preprocessing is clustering (also called segmentation). A cluster is a set of scan points close enough to each other that probably belong to the same object. The clustering method applied here is based on Ref.11. The main idea is to subdivide each scan into small sets of neighboring points (clusters), considering the proximity between two consecutive points of the scan. After clustering, clusters with small number of scan points (smaller than 5) are removed since they are objects far from current vehicle position or noises. Additionally, we analyze the standard deviation ( $\mathrm{x}, \mathrm{y}$ direction) of each cluster to check whether it belongs to natural object (like tree leaves). The basic idea is that, unlike man made object which has certain geometric shape, points from natural object are scattered due to its irregular geometric shape and will have high standard deviation values both in $\mathrm{x}$ and $\mathrm{y}$ directions $^{12}$. Fig. 4 shows the clustering and standard deviation analysis results of one laser scan. Only clusters belong to man made objects remain for further processing.

In section 2, we have mentioned that normal direction of scan point is used for determining correct correspondences in ICP algorithm. Point's normal direction is computed by fitting to a neighborhood of points centered at that point ${ }^{13}$. Furthermore, points with large fitting error will be removed since this usually means the normal direction is poorly defined due to high-curvature points or highly noisy regions. Fig. 5 displays the result, where points enclosed by circles are those with large fitting errors.

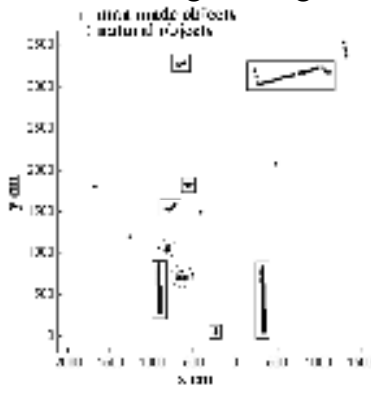

Fig. 4 Clustering and Standard deviation analysis

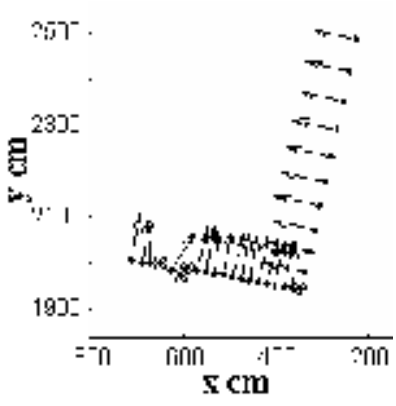

Fig. 5 Normal direction of scan points

\subsection{Map Initialization}

The map is divided into lots of submap, which is called map frame, denoted by $M_{i}=\left\{X_{\mathrm{i}}, S_{\mathrm{i}}\right\} . X_{\mathrm{i}}$ represents vehicle pose and $S_{\mathrm{i}}$ represents laser scan data after preprocessing. Suppose there are $n+1$ map frames in the map and thus the entire map can be expressed as Map = $\left\{M_{1}, M_{2}, \ldots, M_{i}, \ldots, M_{\mathrm{n}}\right\}$. Laser scan frame rate is important and can be set according to the vehicle speed to keep a suitable overlapping size between consecutive laser scans. This overlapping redundancy is essential to carry out following map optimization.

The main difficulty in creating a map is how to get vehicle poses. This is an ego-motion estimation problem ${ }^{10}$. Here, we simply apply odometry based deadreckoning to estimate vehicle poses. However, large accumulative error will be introduced over long time. Therefore, we need optimization approach to improve map accuracy. 
After vehicle poses has been estimated (may include large errors), we can group each vehicle pose with corresponding laser scan data.

\subsection{Map Optimization}

In Fig. 6, suppose curve $\mathrm{AB}$ is true trajectory of vehicle moved in GPS signal block area. Because of accumulated errors in dead-reckoning, the vehicle pose trajectory estimated in map initialization may be like curve $\mathrm{AB}^{\prime}$, which will result in large errors in a global map and make map matching based localization unsuccessful. So we need to improve vehicle pose accuracy to obtain a precise map.

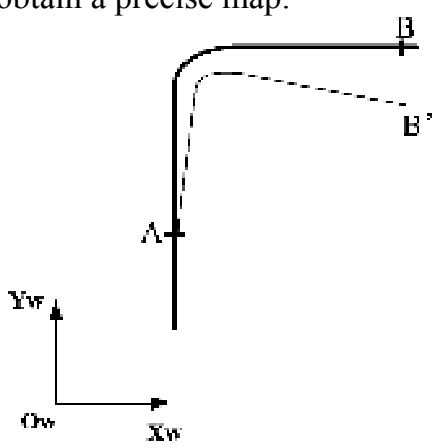

Fig. 6 Vehicle trajectory error in initial map

Some methods have been presented to improve map accuracy. Zhao ${ }^{14}$ first incrementally created a map by sequential matching. Because of error accumulation, there was a gap when closing a loop. Then Zhao distributed error (gap) equally in sequence to obtain a consistent map. This approach is direct and easy to be implemented but the accuracy is limit. Estrada. $\mathrm{C}^{15}$ proposed a nonlinear constrained least-squares optimization approach. The solution was found by Sequential Quadratic Programming (SQP). This is an impressive method for efficient maintenance of loop consistency. However some complicated Jacobians need to be computed. Our map optimization approach was inspired by the approach proposed by $\mathrm{Lu}^{16}$. Nevertheless, we extended his approach to outdoor application and included two types of sensor data from laser radar and GPS (at the entrance and exit points of GPS signal blocked area) in the same optimization framework. In the following, we first explore two kinds of constraints and then linearize the constraints to formulate the definition of optimal linear estimation based map optimization.

\subsubsection{Constraint from range scans registration}

When vehicle moves, the same physical point $\mathrm{p}$ may be observed repeatedly in different vehicle poses, $X_{i}, X_{j}$ for example (the repeatedly observed points are called corresponding points). According to equation (1), we can obtain following equation:

$$
d_{p}=f\left(X_{i}, x v_{i}, y v_{i}\right)-f\left(X_{j}, x v_{j}, y v_{j}\right)=e_{d}
$$

where $e_{d}$ is a small value, representing errors in vehicle poses and laser scan measurements. The above equation indicates constraint on $X_{i}, X_{j}$ by one scan point. If there are $h$ points, constraint can be formulated by minimizing following expression:

$$
\sum_{k=1}^{h}\left\|d_{p}^{k}\right\|^{2}=\sum_{k=1}^{h}\left\|f\left(X_{i}, x v_{i}^{k}, y v_{i}^{k}\right)-f\left(X_{j}, x v_{j}^{k}, y v_{j}^{k}\right)\right\|^{2}
$$

Unfortunately, because of non-linear function $f$, the constraint is also non-linear. In order to formulate a linear map optimization algorithm, the constraint should be linearized.

Let $X_{i}^{\wedge}\left(\hat{x_{i}}, \hat{y_{i}}, \hat{r_{i}}\right)$ and $\hat{X_{j}}\left(\hat{x}, \hat{y_{j}}, \hat{r_{j}}\right)$ be some initial estimates of $X_{i}$ and $X_{j}$ with following equations:

$$
\begin{aligned}
& X_{i}=X_{i}^{\wedge}-\delta X_{i} \\
& X_{j}=X_{j}^{\wedge}-\delta X_{j}
\end{aligned}
$$

Measurement equation of linearized constraint is given by:

$$
D_{i, j}=H_{i} \delta X_{i}-H_{j} \delta X_{j}
$$

The observation of $D_{i, j}$ and corresponding covariance matrix can also be derived, which are denoted by $D_{i, j}{ }^{*}$ and $C_{i, j}{ }^{*}$.

Note that corresponding points used in formulating above constraint are determined by pairwise range scans registration described in section 2 . Since one map frame can carry out valid pairwise registration with several other map frames (here "valid" means registration result don't meet the conditions described in the end of section 2 ), we can obtain a constraints network as displayed in Fig. 7.

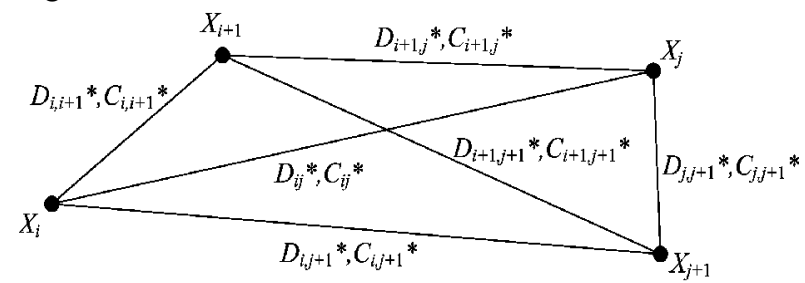

Fig. 7 Constraints network 


\subsubsection{Constraint from GPS data}

In GPS signal blocked area, GPS data is unavailable. However, at the entrance and exit points of this area, GPS data can be used to improve map accuracy. Two vehicle poses corresponding to these entrance and exit points are $X_{0}$ and $X_{\mathrm{n}}$ in map frames $M_{0}$ and $M_{\mathrm{n}}$. Pose relations with these two vehicle poses can be derived as:

$$
T_{0, n}=g\left(X_{0}, X_{n}\right)=(t x, t y, t r)
$$

where:

$$
\left\{\begin{array}{l}
t x=\left(x_{n}-x_{0}\right) \cdot \cos \left(r_{0}\right)+\left(y_{n}-y_{0}\right) \cdot \sin \left(r_{0}\right) \\
t y=-\left(x_{n}-x_{0}\right) \cdot \sin \left(r_{0}\right)+\left(y_{n}-y_{0}\right) \cdot \cos \left(r_{0}\right) \\
t r=r_{n}-r_{0}
\end{array}\right.
$$

Suppose the pose relation obtained by GPS data is $T_{G P S}$, so we can formulate constraint as:

$$
T_{G P S}-T_{0, n}=e_{T}
$$

where $e_{T}$ is a small value, representing errors with GPS data. This constraint is also a non-linear one.

Let $X_{0}^{\wedge}\left(\hat{x_{0}}, \hat{y_{0}}, \hat{r_{0}}\right)$ and $X_{n}^{\wedge}\left(\hat{x_{n}}, \hat{y_{n}}, \hat{r_{n}}\right)$ be initial estimates of $X_{0}$ and $X_{\mathrm{n}}$ with following equations:

$$
\begin{aligned}
& X_{0}=X_{0}^{\wedge}-\delta X_{0} \\
& X_{n}=X_{n}^{\wedge}-\delta X_{n}
\end{aligned}
$$

Measurement equation of linearized constraint is given by:

$$
D_{n, 0}=H_{n} \delta X_{n}-H_{0} \delta X_{0}
$$

The observation of $D_{n, 0}$ and corresponding covariance matrix can also be derived, which are denoted by $D_{n, 0}{ }^{*}$ and $C_{n, 0}{ }^{*}$. The result is the same with that of pairwise range scans registration constraint. Therefore, we can formulate a uniform map optimization framework.

\subsubsection{Map optimization with linear constraints}

Combining all linearized constraints from pairwise range scans registration and GPS data, map optimization can be formulated in an optimal linear estimation sense based on maximum likelihood criterion, which is to minimize the following Mahalanobis distance:

$$
E=\sum_{0 \leq i<j \leq n}\left(D_{i, j}^{*}-D_{i, j}\right)^{T}\left(C_{i, j}^{*}\right)^{-1}\left(D_{i, j}^{*}-D_{i, j}\right)
$$

Above equation can be rewritten as:

$$
E=\sum_{0 \leq i<j \leq n}\left(D_{i, j}^{*}-\left(V_{i}-V_{j}\right)\right)^{T}\left(C_{i, j}^{*}\right)^{-1}\left(D_{i, j}^{*}-\left(V_{i}-V_{j}\right)\right)
$$

where $V_{i}=H_{i} \delta X_{i}$. Furthermore, we can represent the above equation in matrix form:

$$
E=\left(D^{*}-G V\right)^{T}\left(C^{*}\right)^{-1}\left(D^{*}-G V\right)
$$

By applying optimal linear estimation theory, the optimal solution for $V$ which minimize $E$ can be obtained:

$$
V^{*}=\left(G^{T}\left(C^{*}\right)^{-1} G\right)^{-1} G^{T}\left(C^{*}\right)^{-1} D^{*}
$$

Separating $V_{i}{ }^{*}$ from $V^{*}$, we can derive following optimal vehicle pose:

$$
X_{i}^{*}=X_{i}^{\wedge}-\delta X_{i}^{*}=X_{i}^{\wedge}-H_{i}^{-1} \cdot V_{i}^{*}
$$

After optimization, map can be updated with new vehicle poses. Note that Taylor expansion based linearization is applied in our map optimization algorithm, in which accuracy of linearization depends on the accuracy of initial estimate of vehicle poses. Therefore, we can iteratively carry out the proposed algorithm with new derived pose estimates to get more accurate result. The iterative strategy converges very fast. Typically it usually takes three or four iterations to converge to the limit of machine accuracy.

\section{Vehicle Localization}

After mapping in GPS signal blocked area, the created map can be shared with other vehicles to localize themselves in this area. Localization is achieved by map matching, which is also a pairwise range scans registration problem between current laser scan and one map frame in the map. A UKF based fusion strategy is also applied to improve reliability. Sensor data utilized in the localization are laser scans and odometry.

\subsection{Vehicle Motion Model}

Fig. 8 illustrates the relationship between vehicle poses at time step $i$ and time step $i+1$. ICR represents Instant Center of Rotation.

Vehicle motion model can be derived from the geometry:

$$
\left[\begin{array}{c}
x_{i+1} \\
y_{i+1} \\
r_{i+1}
\end{array}\right]=\left[\begin{array}{c}
x_{i}+d s \cdot \cos \left(r_{i}+\pi / 2+d r / 2\right) \\
y_{i}+d s \cdot \sin \left(r_{i}+\pi / 2+d r / 2\right) \\
r_{i}+d r
\end{array}\right]
$$

where $(d s, d r)$ represents the odometry data between time step $i$ and $i+1$, which can be obtained from optical encodes fixed on the vehicle. Equation (5) can be used to predict vehicle poses. 


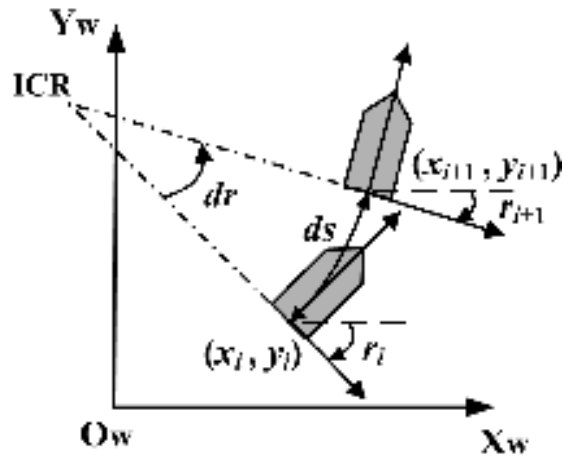

Fig. 8 Vehicle motion model

\subsection{Map Matching Based Localization}

The first problem in map matching is how to choose one best map frame as reference from entire map. To address this problem, we use predicted vehicle pose by equation (5). The map frame whose center is closest to the center of current laser scan in world coordinates is selected. By this idea, the selected map frame is the one who contains the largest part of the same environmental information with the current laser scan, which will improve the reliability of map matching.

Because vehicle pose predicted by odometry is accurate within short period (interval between two successive laser scans), the above idea works well in practice.

Localization is achieved by pairwise registration between the current laser scan (after preprocessing, represented by $S_{\text {cur }}$ ) and the selected reference map frame (represented by $S_{\text {ref }}$ ). Localization mainly includes three steps:

1) Project $S_{\text {ref }}$ to the predicted vehicle pose (by equation (5)), denoted by $S_{\text {ref }}^{*}$.

2) Matching $S_{\text {cur }}$ with $S_{\text {ref }}{ }^{*}$ by pairwise registration described in section 2. The matching result is $(t x, t y$, $t r)$, representing the translation and rotation between two scans.

3) Derive new vehicle pose as:

$$
\left[\begin{array}{l}
x^{*} \\
y^{*} \\
r^{*}
\end{array}\right]=\left[\begin{array}{l}
x \\
y \\
r
\end{array}\right]+\left[\begin{array}{ccc}
\cos (r) & -\sin (r) & 0 \\
\sin (r) & \cos (r) & 0 \\
0 & 0 & 1
\end{array}\right] \cdot\left[\begin{array}{c}
t x \\
t y \\
t r
\end{array}\right]
$$

where $(x, y, r)$ is the predicted vehicle pose.

\subsection{UKF Based Fusion}

Because of noises in data acquisition and errors in preprocessing and ICP registration, localization only by map matching is not reliable. Therefore, we fuse map matching result with odometry based dead-reckoning by $\mathrm{UKF}^{17}$.

UKF is a nonlinear estimation algorithm, which utilizes a deterministic "sampling" approach to calculate the mean and covariance of the state. The state distribution approximated by Gaussian random variables is represented by a set of chosen sample points. These sample points capture the true mean and covariance of the state distribution, and when propagated through the nonlinear system, capture the posterior mean and covariance.

In our approach, system's state is vehicle pose. System's dynamic model is defined as:

$$
\begin{gathered}
X_{i+1}=\left[\begin{array}{c}
x_{i+1} \\
y_{i+1} \\
r_{i+1}
\end{array}\right]=\left[\begin{array}{c}
x_{i}+d s \cdot \cos \left(r_{i}+\pi / 2+d r / 2\right) \\
y_{i}+d s \cdot \sin \left(r_{i}+\pi / 2+d r / 2\right) \\
r_{i}+d r
\end{array}\right]+\left[\begin{array}{l}
q_{1} \\
q_{2} \\
q_{3}
\end{array}\right] \\
Z_{i+1}=\left[\begin{array}{l}
x_{i+1} \\
y_{i+1} \\
r_{i+1}
\end{array}\right]+\left[\begin{array}{c}
v_{1} \\
v_{2} \\
v_{3}
\end{array}\right]
\end{gathered}
$$

where equation (7) is derived from equation (5), representing odometry based dead-reckoning result. $q_{\mathrm{i}}$ represents process noise with dead-reckoning in one time step, which is modeled as Gaussian distribution with zero mean and $C_{q}$ covariance. Measurement equation is expressed by equation (8), given directly by vehicle pose added with noises denoted by $v_{\text {i }}$, which is also modeled as Gaussian distribution with zero mean and $C_{v}$ covariance. Observed measurement value is given by map matching based localization applying equation (6). $C_{q}$ and $C_{v}$ can be roughly estimated offline. If the result of map matching is trustless (meeting the conditions described in the end of section 2), $C_{v}$ should be set to a very large value.

\section{Experiment}

\subsection{Experiments with Synthetic Data}

A simulation system was developed to test and analyze our proposed approach. Fig.9 shows a simple synthetic environment, where polygons represent buildings, circles represent tree trunks and the black triangle represents vehicle. The vehicle can be controlled through keyboard. Configuration of laser radar was set as $80 \mathrm{~m}$ of maximum measurement distance, $0 \sim 180$ 
degree of angular range and 0.5 degree of angular resolution. Moreover, Gaussian noises with zero mean and standard deviation of $4 \mathrm{~cm}$ and standard deviation of 0.2 degree were added to each scan data point to simulate the noises may contained in real scan data.

In the mapping, even small errors introduced in the vehicle pose (especially in the vehicle orientation) may result in large errors in a map. Here, we test the effectiveness of our mapping algorithm. Virtual vehicle was controlled to move along a waved curve and vehicle poses as well as laser scans were recorded for mapping. Additionally, odometry data was also generated by adding Gaussian noise to the relative vehicle poses. The true vehicle trajectory and trajectory by odometry based dead-reckoning are displayed in Fig.10, displaying large accumulative errors with deadreckoning.
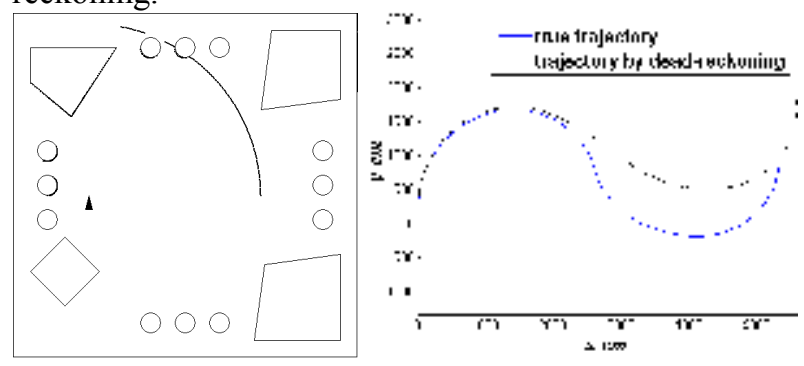

Fig. 9 Synthetic environment

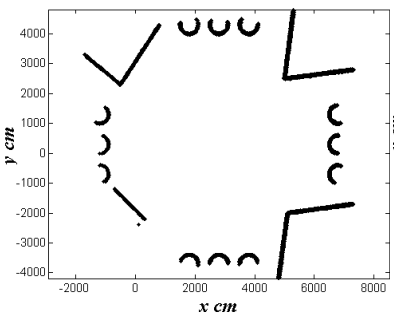

Fig. 11 True map
Fig. 10 Vehicle trajectories

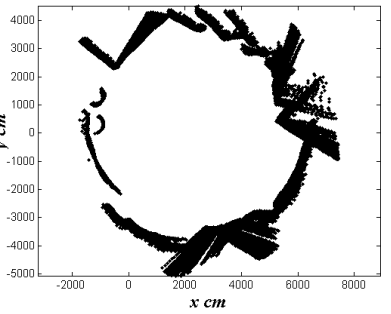

Fig. 12 Noisy map
True map created with true vehicle poses (scan points were transformed and displayed in the world reference frame for a better visualization) is displayed in Fig. 11 and initial noisy map created with vehicle poses by dead-reckoning is in Fig. 12, showing considerable errors in initial map (please see the synthetic environment in Fig. 9 again for a better understanding of the mapping results). Total number of map frames was 154.

Next step, we tested our map optimization algorithm. Totally 906 pairwise scans registration constraints and 1 "GPS data" constraint (by using the

first and last true vehicle poses added with some Gaussian noise to simulate errors of GPS data) were established. The algorithm converges very fast. Typically it takes three or four iterations to converge to the limit of machine accuracy. Fig. 13 displays the map created with the new vehicle poses after map optimization, which is quite similar with the true map. Comparing with Fig.12, our proposed optimization algorithm achieved considerable improvement in map accuracy.

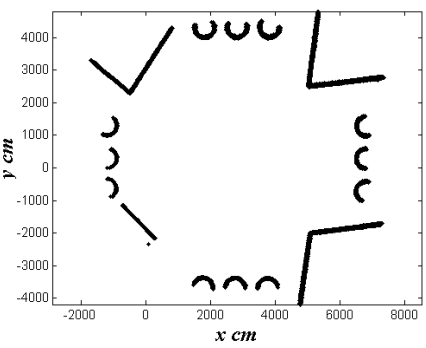

Fig. 13 Optimized map

\subsection{Experiments with Real Data}

The proposed approach has been tested with real data from an intelligent vehicle developed at Shanghai Jiao Tong University. Laser range data was captured by SICK LMS 291-S05, with $80 \mathrm{~m}$ of maximum measurement distance, $0 \sim 180$ degree of angular range and 0.5 degree of angular resolution. Equipped GPS is a NTC2030W RTK-GPS supplied by NavCom company. Odometry data was obtained from two optical encoders equipped on driving motor and steering motor. Resolution of the former encoder is 2000 pulse/rotation and that of the latter encoder is 1000 pulse/rotation. These resolutions were quadrupled by DSP. Vehicle speed was about $2-4 \mathrm{~m} / \mathrm{s}$. The vehicle is shown in Fig. 14

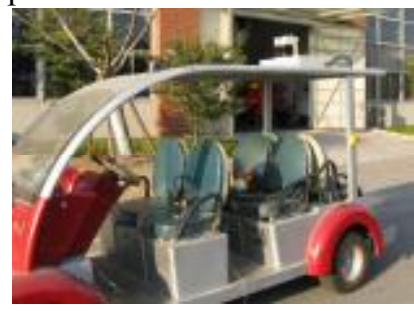

Fig. 14 Intelligent vehicle Fig. $151^{\text {st }}$ experimental place

The first experimental place is displayed in Fig. 15. Vehicle moved along the red path. GPS information during this movement is shown in Fig. 16, where (a) is the number of visible satellites and (b) is GPS working mode (0-fix not available, 1-GPS fix, 2-Differential 
GPS fix, 3-PPS fix, 4-Real Time Kinematic, 5-Float RTK). We used laser scans, odometry data as well as GPS data at "start" and "end" points to create a global map. Laser scans were recorded for mapping about every one meter along the path.

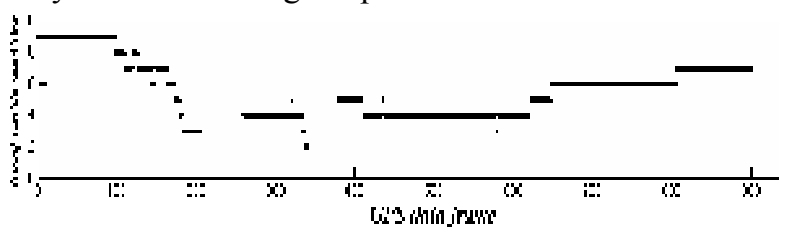

(a) Number of visible satellites

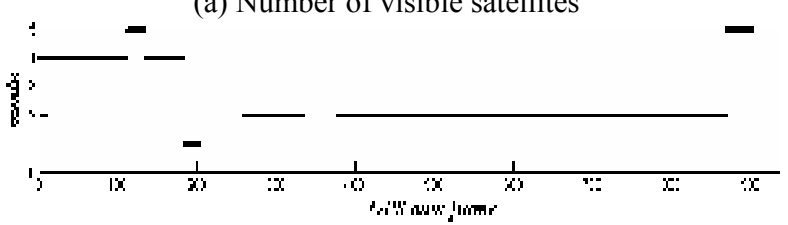

(b) GPS working mode

Fig. 16 GPS information along the path

Initial map without map optimization is displayed in Fig. 17 (scan points were transformed to the world reference frame), which contains 134 map frames. Optimized map created based on constraints from pairwise scans registration (791 constraints) and GPS data is displayed in Fig. 18. Comparing Fig. 17 and Fig. 18 , we can see large improvement in map accuracy by the proposed optimization algorithm.

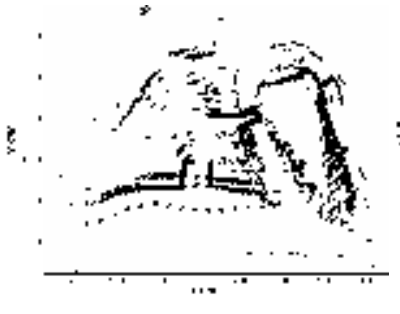

Fig. 17 Initial map

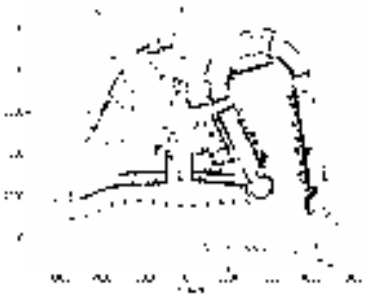

Fig. 18 Optimized map
Second experiment was carried out in the place displayed in Fig.19, where vehicle moved along the red path. GPS information in this experimental place is quite good (almost in RTK or float RTK mode) and we used GPS data as ground truth.

To test our proposed approach, we assumed the region between A and B in Fig. 18 was GPS signal blocked area. Vehicle localization in this area should be achieved by map matching. We used laser scans (every one meter along the path), odometry data as well as GPS data at A and B to create a map. Entire map contains 126 map frames. Initial map without map optimization is displayed in Fig. 20. Optimized map created by using pairwise scans registration constraints (812 constraints) and GPS data constraint is displayed in Fig. 21, showing good improvement in map accuracy.

After a map has been created, localization can be achieved by map matching. Fig.22 shows the localization results. Ground truth is the trajectory by GPS data fused with odometry. These results prove good performance of the proposed approach.

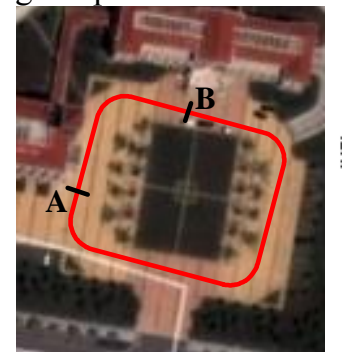

Fig. $192^{\text {nd }}$ experimental place

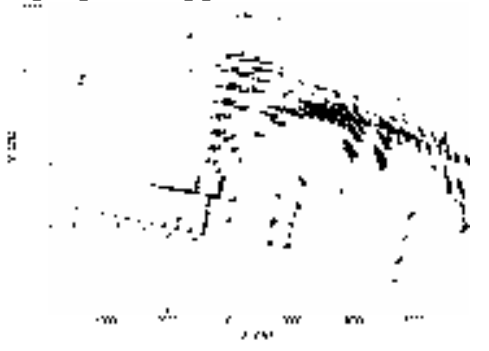

Fig. 20 Initial map

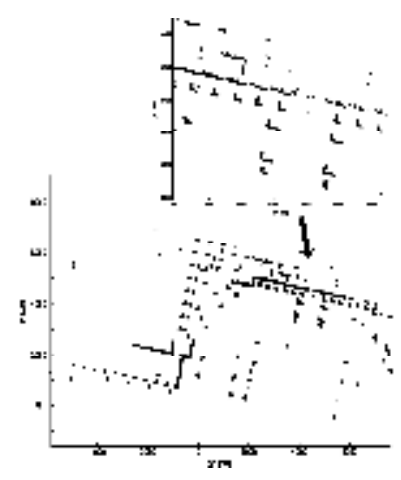

Fig. 21 Optimized map of the second experiment

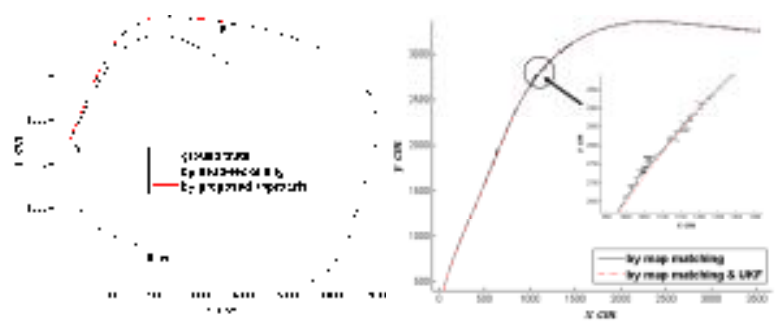

Fig. 22 localization results Fig. 23 Effectiveness

We also compared map matching result with that of map matching \& UKF, displayed in Fig. 23. Because of the noises in measurements and pairwise registration, localization only by map matching was not smooth enough. However, map matching fused with odometry by UKF gave a more reliable result, showing good effectiveness of the proposed fusion strategy. 


\section{Conclusion}

GPS-based approach is popular in vehicle localization. However this approach is sensitive to surrounding environmental conditions. Many objects like high-rise buildings, tunnels, overhead roads or even tall trees can block GPS signals seriously, making localization information unavailable. If GPS signal blocked area is small, Inertial Navigation Systems or dead reckoning can be integrated with GPS to provide continuous localization information, whereas if blocked area is large, other techniques have to be applied. This paper proposed a laser radar based map matching approach to address the localization problem in large GPS signal blocked area.

One difficulty in map matching is how to create a precise map. By linearizing constraints from sensor data, we designed an efficient optimal linear estimation based map optimization algorithm, which improves map accuracy greatly.

Localization is achieved by map matching, which is a pairwise range scans registration between current laser scan and one map frame in the map. Because of noises in data acquisition and errors in data preprocessing and pairwise registration, localization only by map matching is not reliable. Therefore, we fuse map matching result with odometry based deadreckoning to improve reliability. Experimental results show the good performance of the proposed approach. This approach can be integrated with existing GPSbased technique to provide continuous localization information.

Future work will mainly focus on the further improvement in reliability and accuracy. Fusing with other sensors, like camera, is also an emphasis in our future work.

\section{References}

1. W. Wang, F. Hou, H. Tan, A Framework for Function Allocation in Intelligent Driver Interface Design for Comfort and Safety, International Journal of Computational Intelligence Systems, 3(5) (2010), pp.531541

2. J. A. Farrell and M. Barth, The Global Positioning System and Inertial Navigation. (New York: McGrawHill, 1999)

3. I. Skog, P. Handel. In-Car Positioning and Navigation Technologies-A Survey. IEEE Transactions on Intelligent Transportation Systems, 10(1), (2009), pp. 421.
4. W. Wang, Y. Mao, J. Jing, Driver's various information process and multi-ruled decision-making mechanism: a fundamental of intelligent driving shaping model, International Journal of Computational Intelligence Systems, 4(3) (2011), pp.297-305.

5. Y. Cui and S. S. Ge, Autonomous vehicle positioning with GPS in urban canyon environments, IEEE Transactions on Robotics and Automation, 19(1) (2003), pp. 15-25.

6. T. H. Chang, L. S. Wang and F. R. Chang. A Solution to the Ill-Conditioned GPS Positioning Problem in an Urban Environment, IEEE Transactions on Intelligent Transportation Systems, 10(1) (2009), pp. 135-145.

7. M. Quddus, W. Ochieng, and R. Noland. Current mapmatching algorithms for transport applications: State-ofthe art and future research directions. Transportation research. Part C, Emerging Technologies, 15(5) (2007), pp.312-328.

8. D. Lee, W. Chung and M. Kim. A reliable position estimation method of the service robot by map matching. In the Proceeding of IEEE International Conference on Robotics and Automation, (2003), pp.2830 2835.

9. G. Giorgio, et al. Fast and Accurate SLAM with RaoBlackwellized Particle Filters. Robotics and Autonomous Systems. 55(1) (2007), pp.30-38.

10. M. Yang, et al. Laser radar based real-time ego-motion estimation for intelligent vehicles. In the Proceedings of IEEE Intelligent Vehicle Symposium. (2002), pp.44-51.

11. K. C. J.Dietmayer, J. Sparbert and D. Streller. Model Based Object Classification and Object Tracking in Traffic Scenes from Range Images. In the Proceedings of IEEE Intelligent Vehicle Symposium (2001), pp.25-30.

12. D. Manandhar, R. Shibasaki. Vehicle-borne laser mapping system (VLMS) for 3-D GIS. In the Proceedings of Geoscience and Remote Sensing Symposium. vol.5 (2001), pp.2073-2075.

13. F. Lu, E. Milios. Robot Pose Estimation in Unknown Environments by Matching 2D Range Scans. Journal of Intelligent and Robotic Systems. 18(3) (1997) pp.249-275.

14. H. Zhao, R. Shibasaki, Reconstructing urban 3D model using vehicle-borne laser radars. Proceedings of Third International Conference on 3-D Digital Imaging and Modeling (2001), pp.349-356.

15. C. Estrada, J. Neira, and J. D. Tardos. Hierarchical SLAM: Real-Time Accurate Mapping of Large Environments. IEEE Transactions on Robotics. 21(4) (2005), pp.588-596.

16. F. Lu, E. Milios. Globally Consistent Range Scan Alignment for Environment Mapping. Autonomous Robots, 4(4) (1997), pp.333-349.

17. S. J. Julier and J. K. Uhlmann. Unscented Filtering and Nonlinear Estimation. In the Proceeding of IEEE. Vol.92 (2004), pp. 401-422. 